Rev Inv Vet Perú 2015; 26(1): 1-8

http://dx.doi.org/10.15381/rivep.v26i1.10915

ArTículo de Revisión

\title{
Cortisol como Indicador Fiable del Estrés en Alpacas y Lamas
}

\author{
Cortisol as a Reliable Indicator of Stress in Alpacas and Llamas \\ Nino Arias ${ }^{1,2,3}$, Billie Velapatiño ${ }^{1}$
}

\section{Resumen}

Se revisan investigaciones sobre medición de cortisol y sus metabolitos en alpacas y llamas. Las concentraciones de cortisol en muestras de plasma, suero y saliva, así como de metabolitos de cortisol en muestras de heces, se elevan luego de situaciones de estrés. La interpretación de estos resultados debe complementarse con otros signos fisiológicos y observaciones del comportamiento.

Palabras clave: bienestar animal; camélidos; comportamiento; cortisol; estrés

\section{Abstract}

Research papers on the measurement of cortisol and its metabolites in alpacas and llamas were reviewed. Plasma, serum, saliva cortisol and fecal cortisol metabolites concentrations increase after stress events. The interpretation of these results should be complemented with additional physiological signs and behavioral observations.

Key words: animal welfare; behaviour; camelids; cortisol; stress

\footnotetext{
${ }^{1}$ Facultad de Veterinaria y Zootecnia, Universidad Peruana Cayetano Heredia, Lima, Perú

${ }^{2}$ Animal Welfare Program, Faculty of Land and Food Systems, University of British Columbia, Canada

${ }^{3}$ E-mail: nino.arias@upch.pe
}

Recibido: 22 de abril de 2014

Aceptado para publicación: 29 de setiembre de 2014 


\section{INTRODUCCIÓN}

El criador de alpacas (Vicugna pacos) y llamas (Lama glama) pone énfasis en la adecuada alimentación y salud de sus animales para lograr el mayor rendimiento productivo. El manejo de los animales en un ambiente de confort, higiene y sin estrés ni dolor permite que puedan manifestar su esperada conducta social y maximizar su rendimiento productivo y reproductivo (von Keyserlingk et al., 2009).

El estrés es la respuesta del organismo frente a los estímulos (estresores) que alteran su homeostasis. Como respuesta a estos estímulos, se dispara una cadena de respuestas fisiológicas dirigidas por el cerebro que comprenden cambios en la conducta, en la respuesta inmune, en la activación del sistema nervioso autónomo (incremento de la frecuencia cardiaca) y en la activación del eje hipotálamo-pituitario-adrenal (HPA). El eje HPA libera la hormona cortisol en la sangre circulante. Una respuesta rápida de estrés es buena porque permite al animal superar cualquier adversidad de su medio ambiente (por ejemplo, escapar de un predador), pero se afecta la salud y bienestar del animal cuando el estímulo estresor es prolongado (para una revisión detallada, ver Dickens et al., 2010).

Después del evento estresante, el sistema nervioso estimula un grupo de neuronas hipofisiotrópicas ubicadas en el hipotálamo, donde se liberan en la circulación portal las hormonas liberadoras de corticotropina (CRH) y arginina-vasopresina (AVP), que a su vez estimulan la liberación de la hormona adrenocorticotropa (ACTH) en la circulación sanguínea. En respuesta, la corteza adrenal produce glucocorticoides (cortisol, corticosterona) (Herman et al., 2003).

La respuesta al estrés incluye incrementos de la concentración de cortisol en sangre y aumento de la actividad cardiovascular y cognitiva, así como la movilización de gluco- sa, y mientras tanto se suprime o minimiza la función reproductiva, inmune y digestiva (Dallman, 2003). El cortisol circulante en sangre es metabolizado por el hígado y excretado en la orina y heces. En el caso de los ovinos, las heces contienen diversos grupos de metabolitos del cortisol, pero solo trazas del cortisol en sí (Palme y Möstl, 1997).

Algunas actividades cotidianas de la crianza de alpacas y llamas son estresoras y sus consecuencias están asociadas a abortos y ocurrencia de enfermedades. Knight et al. (1995) reporta la pérdida de fetos asociada al estrés de dos horas de transporte y posterior reubicación a un nuevo ambiente de 16 alpacas con cerca de siete meses de gestación. Del mismo modo, la esquila o el transporte se asocia a casos de septicemia de la bacteria comensal Streptococcus equi subespecie zooepidemicus. Esta bacteria produce una peritonitis y pleuritis fibrinopurulenta, también llamada «fiebre de la alpaca» (Cebra et al., 2000; Hewson y Cebra, 2001). Asimismo, en un grupo de alpacas en los Estados Unidos que estuvo expuesto a diversos estresores (parasitismo, lesiones o enfermedades), se demostró que aquellas que fueron esquiladas hasta $2 \mathrm{~cm}$ de la piel soportaban mejor el calor del verano $\left(20\right.$ a $30^{\circ} \mathrm{C}$ ) en comparación con las alpacas que no fueron esquiladas (Navarre et al., 2001).

La presente revisión se enfoca en el uso del cortisol como indicador de estrés en alpacas y llamas.

\section{Cortisol en Sangre}

En los estudios iniciales, al inyectarse ACTH a un grupo de alpacas para estimular la producción de glucocorticoides en la corteza adrenal, se identificó a la corticosterona como el principal glucocorticoide, encontrándose una gran variación entre individuos (Guerra-García et al., 1975). Asimismo, Crossley et al. (1994) reportaron altos niveles de cortisol plasmático en alpacas del altiplano chileno antes y el día posterior al transporte 
hacia una estación cercana al nivel del mar, indicando que los niveles altos de cortisol antes del viaje podrían deberse al ambiente hostil del altiplano desde donde fueron transportadas y que los niveles de cortisol disminuyeron cuando las alpacas se adaptaron a un ambiente con mejor calidad de alimento disponible.

En otro estudio, donde se evaluó el estrés causado por 30 minutos de transporte en alpacas, se encontró que la concentración de cortisol estaba elevada inmediatamente después del transporte, pero a las cuatro horas de reposo disminuyeron hasta niveles similares al momento previo al transporte. Asimismo, el ritmo cardiaco no cambió y el comportamiento no estuvo asociado al incremento de la concentración de cortisol (Anderson et al., 1999b).

En el Perú, las crías de alpaca son separadas de sus madres al inicio de la primavera (setiembre a octubre), cuando tienen entre 7 y 8 meses de edad. En este contexto, Bravo et al. (2001) encontraron que las crías de alpaca tienen concentraciones elevadas de cortisol durante los tres días posteriores al destete y disminuyen al quinto día.

\section{Cortisol en Saliva}

La punción de la aguja para obtener la muestra de sangre es un estresor en la vaca (Hopster et al., 1999), al igual que en otras especies. No obstante, en la alpaca y la llama se ha podido observar que repetidas punciones a la vena no llegaron a influir en los niveles de cortisol (Anderson et al., 1999a). De todas formas, el estrés de la punción puede ser evitado mediante la recolección de muestras de saliva, especialmente si el animal ha sido entrenado para aceptar abrir la boca y tolerar por unos segundos el material absorbente que recogerá la saliva. Esta metodología permitió la evaluación del estrés de transporte en caballos (Schmidt et al., 2010).
El cortisol circulante en la sangre se une a las globulinas fijadoras de corticoesteroides (transcortina, albúmina). Los cambios de concentración de estas proteínas influyen en la concentración de cortisol en la sangre (ElBahr et al., 2006). El cortisol pasa a la saliva en la glándula parótida por difusión simple (Umeda et al., 1981) y, de este modo, se puede medir el cortisol libre (no unido a proteínas) en muestras de saliva. La muestra se obtiene fácilmente introduciendo un algodón en la boca de alpacas y llamas por un lapso de 3 a 5 minutos, tiempo suficiente para que ocurra la saturación del algodón con saliva (Anderson et al., 1999a).

La concentración de cortisol se eleva significativamente en el suero a consecuencia de 30 minutos de transporte (Anderson et al., 1999b). Sin embargo, en el caso de las muestras de saliva y bajo condiciones experimentales similares, la concentración de cortisol en saliva se elevó solamente en la muestra tomada cuatro horas posteriores al transporte (Anderson et al., 1999a).

\section{Metabolitos de Cortisol en Heces}

Palme y Möstl (1997) desarrollaron el inmunoanálisis enzimático (EIA) 11oxoetiocolanolona con anticuerpos que reaccionan contra los metabolitos de cortisol que tienen la estructura 11,17-dioxoandrostano. Este EIA se validó en camélidos sudamericanos, a la vez que se evaluó el estrés debido al transporte en llamas y al «chaccu» en vicuñas (Arias et al., 2013). Esta metodología es adecuada para la evaluación del estrés en varias especies rumiantes (Palme, 2012).

En la Figura 1 se muestra el pico en la concentración de cortisol en plasma luego de la estimulación con ACTH. A continuación se observa su reflejo con otro pico en la concentración de metabolitos de cortisol en heces. El tiempo que separa ambos picos está determinado por el tiempo de pasaje intesti- 


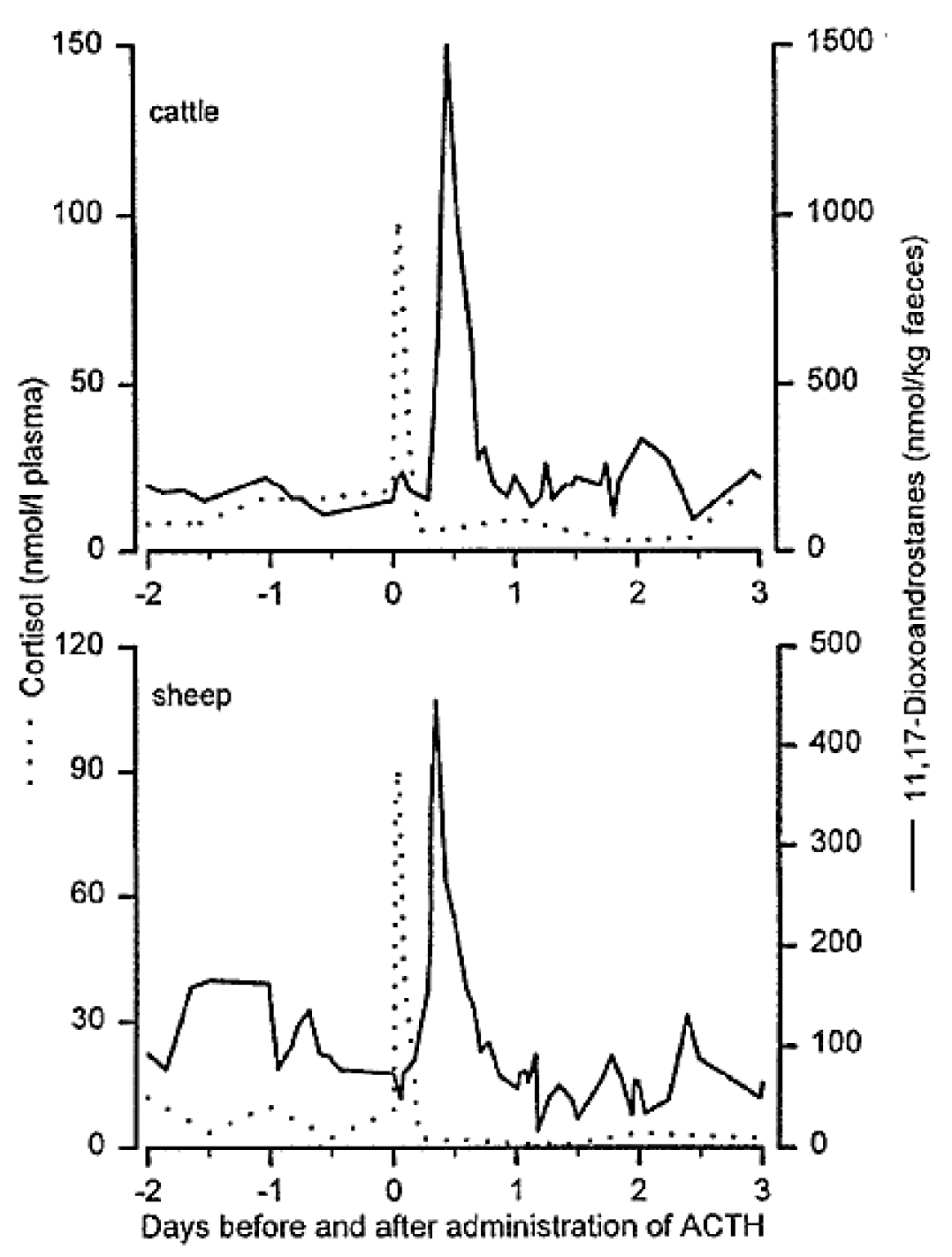

Figura 1. Cinética de las concentraciones de cortisol en sangre (nmol/l) y metabolitos de cortisol (11,17-dioxoandrostanos) en heces ( $\mathrm{nmol} / \mathrm{kg}$ ) dos días antes y tres días después de la administración de ACTH en una vaca (panel superior) y una oveja (panel inferior). Tomado de: Palme et al. (1999). Vet Med Austria 86: 237-241. Con permiso de $\ddot{O} G T$.

nal del duodeno al recto (Palme et al., 1996), donde el tiempo medio de pasaje intestinal del duodeno al recto en alpacas y llamas es de 28 y 33 horas, respectivamente (Arias $e t$ al., 2013).

En el caso de muestras fecales, es importante tomar en cuenta que las muestras deben ser recolectadas tan pronto como ocu- rra la defecación y homogeneizarse en la misma bolsa que fueron recogidas para prevenir variación de concentraciones de metabolitos dentro de la muestra. Asimismo, las muestras deben ser congeladas de inmediato a $-20{ }^{\circ} \mathrm{C}$ para prevenir la degradación de los esteroides por la actividad enzimática de las bacterias presentes en las heces (Bokkenheuser et al., 1978; Möstl et al., 1999; Arias et al., 2013). 
Otros factores que podrían influir en la medición de metabolitos fecales de cortisol son el estado reproductivo y sexo del animal, así como la estación del año (Palme, 2012). Además, es posible que la hipoxia afecte la producción de cortisol, lo cual podría ser medido mediante estimulación de la corteza adrenal de un mismo grupo de animales en la altitud y al nivel del mar (Panesar, 2004).

\section{Inmunoanálisis de Cortisol}

Al momento, no ha sido validado un kit comercial para medir concentraciones de cortisol en muestras de suero o plasma provenientes de alpacas y llamas. En este sentido, la validación técnica de un kit de diagnóstico usado para otras especies incluye determinar su sensibilidad y su especificidad. Se deberán usar como referencia una muestra control positiva (estándar analítico de cortisol, CAS 50-23-7) y otra muestra control negativa ( sin cortisol). Asimismo, se deberá ensayar un control externo, agregando estándar analítico de cortisol en diluciones seriadas a muestras de suero, plasma o saliva de camélido saludable y sin estrés, y procesarlas de acuerdo con las instrucciones del kit. Este procedimiento permitirá detectar inhibidores en la muestra, así como la capacidad de detección de cortisol de la prueba (sensibilidad técnica). También es importante evaluar posibles reacciones cruzadas a fin de investigar la capacidad de la prueba para distinguir entre estructuras parecidas al cortisol (hormonas esteroideas como estradiol, testosterona, etc.) (Ver metodología de laboratorio en Scheel et al., 2006).

Además de la validación analítica del kit, es necesario realizar la validación fisiológica, lque se hace mediante la inyección de ACTH. Se espera medir un súbito incremento y luego la disminución de cortisol a los valores iniciales. Esto debe realizarse para cada especie a ser investigada. Las comparaciones de valores esperados bajo diferentes situaciones de estrés, edades, sexo o especie solamente son posibles si se utiliza la misma metodolo- gía de laboratorio o el mismo kit comercial de inmunoanálisis. En consecuencia, no es posible obtener valores referenciales y el punto de corte para discriminar situaciones de estrés y sin estrés; sin embargo, las situaciones estresantes pueden compararse, siempre y cuando se midan por el mismo método (Palme, 2012).

Morrow et al. (2002) validaron un kit comercial de radioinmunoanálisis (RIA Corticosterona ${ }^{125}$ I, MP Biomedicals, EEUU) para bovinos. Este kit está diseñado por el fabricante para su uso en ratas y ratones. La reacción cruzada de anticuerpos favorece en este caso la medición de algunos metabolitos (Palme, 2012).

\section{Monitoreo del Comportamiento}

El animal enfermo deja de comer, se aparta del grupo y pasa más tiempo en reposo. Este sufrimiento indeseado está asociado a las citoquinas que se producen durante la respuesta a la enfermedad y contribuyen a la supervivencia del animal (Gregory, 1998). Por ejemplo, las vacas con metritis severa comen con menos frecuencia que sus compañeras saludables; pero además se determinó que estas vacas reducían el tiempo empleado en su alimentación una semana antes del parto en comparación con las vacas saludables (Huzzey et al., 2007). Un cambio en la conducta de alimentación es un potencial indicador para la detección temprana de esta enfermedad.

Respecto a la observación de la conducta de pastoreo, se observó menor consumo de alimento en alpacas confinadas en soledad (Pollard y Littlejohn, 1995). Del mismo modo, se observó que las alpacas pasan más tiempo pastoreando en la pradera alto andina en comparación con las zonas bajas (Raggi et al., 1994).

En la Figura 2 se muestra un caso donde a mayor tiempo de duración del estrés debido al confinamiento de guanacos, mayor 


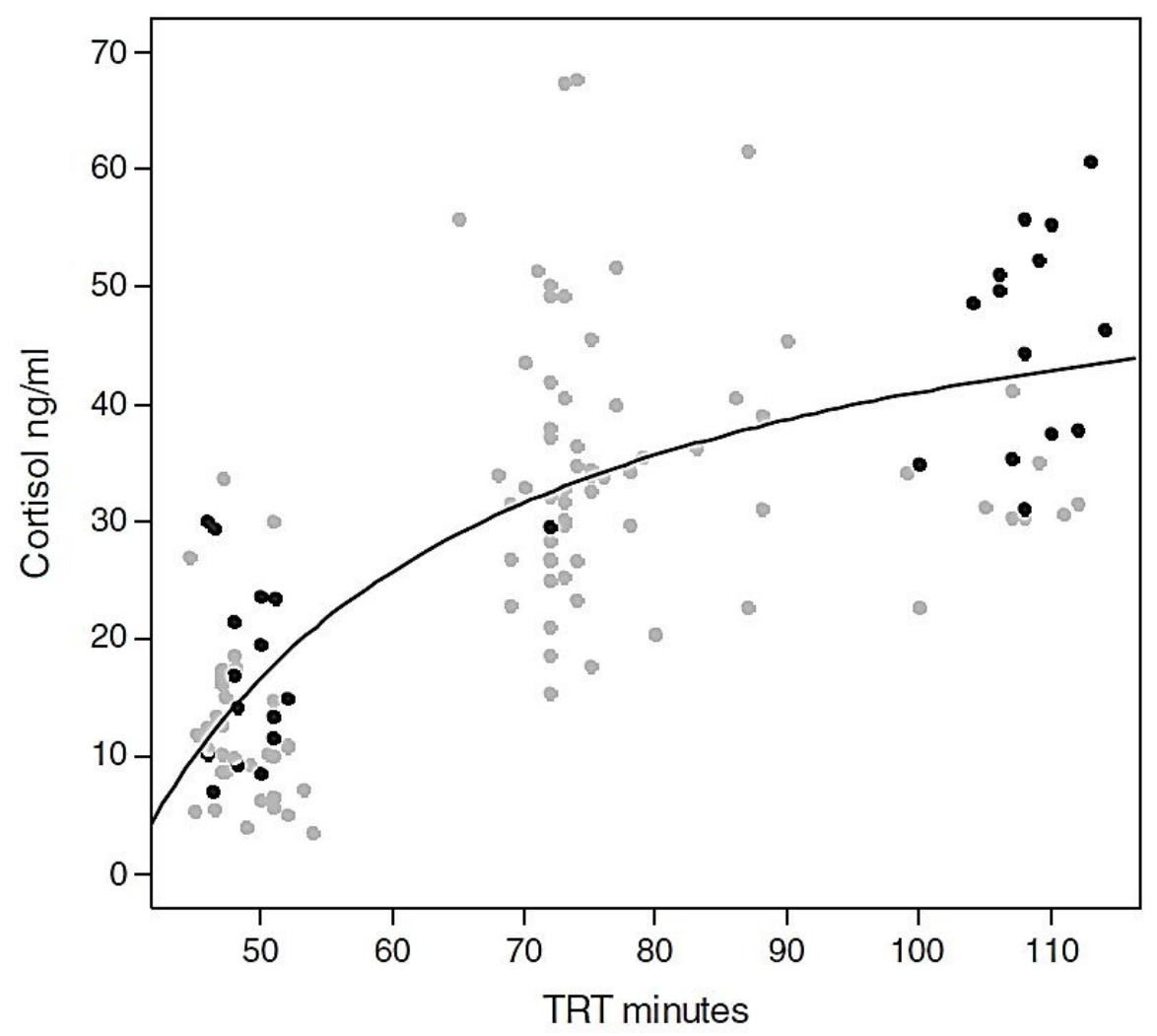

Figura 2. Correlación entre el tiempo total de retención de guanacos (TRT, en minutos) y la concentración de cortisol en la sangre de los mismos guanacos (ng/ml). Círculos negros: representan guanacos hembras; círculos grises: representan guanacos machos. Tomado de Carmanchahi et al. (2011). Wildlife Research 38: 61-68. Con permiso de CSIRO Publishing. Ver http://www.publish.csiro.au/nid/144/paper/ WR10170.htm

será la concentración de cortisol en su sangre. Carmanchahi et al. (2008) observaron una correlación positiva entre el tiempo de manipulación de guanacos y la concentración de cortisol en sangre. El tiempo de retención de cada animal incluyó su captura, su esquila, la extracción de sangre y su liberación.

\section{Conclusiones}

Se concluye que la medición de cortisol en sangre y metabolitos de cortisol $(11,17$ dioxoandrostanos) en heces son indicadores fiables para detectar si la alpaca o llama ha estado expuesta a un evento estresante.

\section{Agradecimiento}

El estudio fue financiado por la Beca de Investigación Post Doctoral del Consejo Nacional de Ciencia, Tecnología e Innovación Tecnológica del Perú.

\section{Literatura CitadA}

\section{Anderson DE, Silveira F, Grubb T.} 1999a. Effects of venipuncture and correlation of plasma, serum and saliva cortisol concentration with transportation stress in camelids. J Camel Pract Res 6: 249-254. 
2. Anderson DE, Grubb T, Silveira F. 1999b. The effect of short duration transportation on serum cortisol in alpacas (Lama pacos). Vet J 157: 189191.

3. Arias N, Requena M, Palme R. 2013. Measuring faecal glucocorticoid metabolites as a non-invasive tool for monitoring adrenocortical activity in South American camelids. Anim Welfare 22: 2531. doi: $10.7120 / 09627286.22 .1 .025$

4. Bravo PW, Garnica J, Aviles E. 2001. Cortisol concentrations in the perinatal and weaning periods of alpacas. Anim Reprod Sci 67: 125-129. doi: 10.1016/ S0378-4320(01)00112-9

5. Bokkenheuser VD, Winter J, Kelly WG 1978. Metabolism of biliary steroids by human fecal flora. Am J Clin Nutr 31: S221-S226.

6. Carmanchahi PD, Ovejero R, Marull C, López GC, Schroeder N, Jahn GA, et al. 2011. Physiological response of wild guanacos to capture for live shearing. Wildlife Res 38: 61-68. doi: 10.1071/ WR10170

7. Cebra CK, Heidel JR, Cebra ML, Tornquist SJ, Smith BB. 2000. Pathogenesis of Streptococcus zooepidemicus infection after intratracheal inoculation in llamas. Am J Vet Res 61: 1525-1529. doi: 10.2460/ajvr.2000.61.1525

8. Crossley JC, Marín MP, Ferrando G, Raggi LA. 1994. Modificaciones adaptativas de algunas constantes fisiológicas de alpaca (Lama pacos) sometidas a cambios de ambiente. Arch Zootec 43: 215-223.

9. Dallman MF. 2003. Stress by any other name...? Horm Behav 43: 18-20. doi: 10.1016/S0018-506X(02)00034-X

10. Dickens MJ, Delehanty DJ, Romero LM. 2010. Stress: an inevitable component of animal translocation. Biol Conserv 143: 1329-1341. doi: 10.1016/ j.biocon.2010.02.032

11. El-Bahr SM, Kahlbacher H, Patzl M, Palme RG. 2006. Binding and clearance of radioactive adrenaline and noradrenaline in sheep blood. Vet Res Commun
30: 423-432. doi: 10.1007/s11259-0063244-1

12. Gregory NG. 1998. Physiological mechanisms causing sickness behaviour and suffering in diseased animals. Anim Welfare 7: 293-305.

13. Guerra-García R, Coyotupa J, Losno W, Sumar J. 1975. Estudios de la función adrenal en la alpaca. En: VI Congreso Peruano de Endocrinología. Cajamarca: Sociedad Peruana de Endocrinología.

14. Herman JP, Figueiredo H, Mueller NK, Ulrich-Lai Y, Ostrander MM, Choi DC, Cullinan WE. 2003. Central mechanisms of stress integration: hierarchical circuitry controlling hypothalamo-pituitary-adrenocortical responsiveness. Front Neuroendocrinol 24: 151-180. doi: 10.1016/j.yfrne.2003.07.001

15. Hewson J, Cebra CK. 2001. Peritonitis in a llama caused by Streptococcus equi subspecies zooepidemicus. Can Vet J 42: 465-467.

16. Hopster H, van der Werf JTN, Erkens JH, Blokhuis HJ. 1999. Effects of repeated jugular puncture on plasma cortisol concentrations in loose-housed dairy cows. J Anim Sci 77: 708-714.

17. Huzzey JM, Veira DM, Weary DM, von Keyserlingk MA. 2007. Prepartum behavior and dry matter intake identify dairy cows at risk for metritis. J Dairy Sci 90: 3220-3233. doi: 10.3168/jds.2006807

18. Knight TW, Ridland M, Scott I, Death AF, Wyeth TK. 1995. Foetal mortality at different stages of gestation in alpacas (Lama pacos) and the associated changes in progesterone concentrations. Anim Reprod Sci 40: 89-97. doi: 10.1016/ 0378-4320(95)01415-V

19. Morrow CJ, Kolver ES, Verkerk GA, Matthews LR. 2002. Fecal glucocorticoid metabolites as a measure of adrenal activity in dairy cattle. Gen Comp Endocrinol 126: 229-241. doi: 10.1006/ gcen.2002.7797

20. Möstl E, Messmann S, Bagu E, Robia C, Palme R. 1999. Measurement of glucocorticoid metabolite concentrations 
in faeces of domestic livestock. J Vet Med A46: 621-631. doi: 10.1046/j.14390442.1999.00256.x

21. Navarre CB, Heath AM, Wenzel J, Simpkins A, Blair E, Belknap E, Pugh DG. 2001. A comparison of physical examination and clinicopathologic parameters between sheared and nonsheared alpacas (Lama pacos). Small Ruminant Res 39: 11-17. doi: 10.1016/S0921-4488(00)00176-0

22. Palme R, Fischer P, Schildorfer $H$, Ismail MN. 1996. Excretion of infused ${ }^{14} \mathrm{C}$-steroid hormones via faeces and urine in domestic livestock. Anim Reprod Sci 43: 43-63. doi: 10.1016/03784320(95)01458-6

23. Palme R, Möstl E. 1997. Measurement of cortisol metabolites in faeces of sheep as a parameter of cortisol concentration in blood. Z Saugetierkd 62(Suppl 2): 192-197.

24. Palme R, Robia C, Messmann S, Hofer J, Möstl E. 1999. Measurement of faecal cortisol metabolites in ruminants: a non-invasive parameter of adrenocortical function. Wien Tierarztl Monatsschr 86: 237-241.

25. Palme R. 2012. Monitoring stress hormone metabolites as a useful, noninvasive tool for welfare assessment in farm animals. Anim Welfare 21: 331-337. doi: 10.7120/09627286.21.3.331

26. Panesar N. 2004. High altitude sickness. Is acute cortisol deficiency involved in its pathophysiology? Med Hypotheses 63: 507-510. doi: 10.1016/ j.mehy.2003.11.039
27. Pollard JC, Littlejohn RP. 1995. Effects of social isolation and restraint on heart rate and behaviour of alpacas. Appl Anim Behav Sci 45: 165-174. doi: 10.1016/0168-1591(95)00588-J

28. Raggi LA, Jiliberto E, Urquieta B. 1994. Feeding and foraging behaviour of alpaca in northern Chile. J Arid Environ 26: 73-77. doi: 10.1006/ jare.1994.1011

29. Scheel CM, Handali S, Bueno EC, Khan A, Hancock K, Gonzalez AE, Garcia HH, et al. 2006. Development of a normal human immunoglobulin $\mathrm{G}$ standard curve for enzyme-linked immunosorbent assay: use for comparison of antigen efficacy. $\mathbf{J}$ Immunoassay Immunochem 27: 173-81. doi: 10.1080/15321810600573028

30. Schmidt A, Möstl E, Wehnert C, Aurich J, Müller J, Aurich C. 2010. Cortisol release and heart rate variability in horses during road transport. Horm Behav 57: 209-215. doi: 10.1016/ j.yhbeh.2009.11.003

31. von Keyserlingk MAG Rushen J, de Passille AM, Weary DM. 2009. The welfare of dairy cattle - Key concepts and the role of science. J Dairy Sci 92: 4101-4111. doi: 10.3168/jds.2009-2326

32. Umeda T, Hiramatsu R, Iwaoka T, Shimada T, Miura F, Sato T. 1981. Use of saliva for monitoring unbound free cortisol levels in serum. Clin Chim Acta 110: 245-253. doi: 10.1016/00098981(81)90353-3 